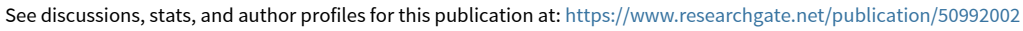

\title{
Establishing Fraction-Decimal Equivalence Using A Respondent-Type Training Procedure
}

Article in The Psychological record · January 2011

DOI: 10.1007/BF03395391 · Source: OAl

\section{CITATIONS}

33

2 authors, including:

Geraldine Leader

National University of Ireland, Galway

94 PUBLICATIONS 2,448 CITATIONS

SEE PROFILE
READS

102

Some of the authors of this publication are also working on these related projects:

GEMMA (Genome, Environment, Microbiome and Metabolome in Autism View project

Application of Applied Behavior Analysis to the Management of Gestational Weight Gain and Investigation into Weight Mediated Conditions as Risk Factors for the Development of Autism Spectrum Disorder in Offspring View project 


\section{ESTABLISHING FRACTION-DECIMAL EQUIVALENCE USING A RESPONDENT-TYPE TRAINING PROCEDURE.}

\author{
GERALDINE LEADER \\ University of Ulster at Jordanstown
}

\author{
DERMOT BARNES-HOLMES \\ National University of Ireland, Maynooth
}

The purpose of this study was to teach children fractiondecimal equivalence using the respondent-type training procedure and test for any emergent generalization. In the first experiment, subjects were respondently trained on the conditional discriminations; $\mathrm{A} 1 \rightarrow \mathrm{B} 1, \mathrm{~A} 2 \rightarrow \mathrm{B} 2$, and tested $\mathrm{B} 1-\mathrm{A} 1, \mathrm{~B} 2-\mathrm{A} 2$. Subjects were then trained on the conditional discriminations $\mathrm{C} 1 \rightarrow \mathrm{B} 1, \mathrm{C} 2 \rightarrow \mathrm{B} 2$ and tested $\mathrm{B} 1-\mathrm{C} 1, \mathrm{~B} 2-\mathrm{C} 2$. Subjects were subsequently tested for the emergence of the untrained relations A-C and C-A. When subjects were presented with the Stimulus A1 they observed $1 / 4$ and when subjects were presented with $A 2$ they observed 2/4. When subjects were presented with B1 they observed a circle with the upper left quarter shaded and when subjects were presented with B2 they observed a circle divided into four quarters with the upper half shaded. When subjects were presented with $\mathrm{C} 1$ they observed 0.25 and when subjects were presented with $\mathrm{C} 2$ they observed 0.50 . Experiment 2 was identical to Experiment 1 except that subjects were administered three generalization tests. In Generalization Test Number 1, the Stimuli $A 1, A 2, C 1$, and C2 served as samples and shapes not seen in training but with the same shaded areas served as comparison stimuli. Generalization Test No. 2 was identical to the previous test, except that the comparison stimuli consisted of a shape not seen in training and the shaded area was altered. Generalization Test No. 3 was identical to the previous two tests except that the comparison stimuli were altered in that the number of shaded and unshaded areas was increased. Experiment 3 was identical to Experiment 2 except that an extra generalization test was added to the experiment. This test was identical to Generalization Test No. 3 of Experiment 2, except that the shaded areas were contiguous with each other.

In Experiment 1, subjects were successful on all equivalence

This research was conducted as part of Geraldine Leader's doctoral research program under the supervision of Dermot Barnes-Holmes. These data were presented at the Symposium on Stimulus Equivalence at the Annual European Meeting of the Experimental Analysis of Behavior Group, London, April 1998. Requests for reprints may be addressed to Geraldine Leader, School of Psychology and Communication Science, University of Ulster at Jordanstown, County Antrim, BT37 0QB, Northern Ireland. (E-mail: G.Leader@ulst.ac.uk). 
tests. In Experiment 2, subjects were successful on all equivalence tests and $50 \%$ of subjects successfully completed the final generalization tests. In Experiment 3, subjects were successful on all equivalence tests and on all generalization tests.

When humans are taught a series of related conditional discriminations, the stimuli that enter into these discriminations often become connected to each other in novel ways not explicitly taught in training. This phenomenon has been investigated using a matching-to-sample procedure, as developed by Murray Sidman $(1971,1980,1986,1987)$. In a typical experiment, subjects are taught to select Stimulus B and Stimulus $C$ (called the comparisons) in the presence of Stimulus $A$ (called the sample). During testing, subjects may select $A$ in the presence of $A$ (reflexivity), $A$ in the presence of both $C$ and $B$ (symmetry), and $C$ in the presence of $B$, and $B$ in the presence of $C$ (combined symmetry and transitivity or an equivalence relation). If these performances are produced in the absence of differential reinforcement, they are normally accepted as evidence that the $\mathrm{A}, \mathrm{B}$, and $\mathrm{C}$ stimuli participate in an equivalence class (see Barnes, 1994; Fields \& Verhave, 1987; Hayes, 1991).

Stimulus equivalence has provided researchers with an efficient and economical means of establishing novel, complex behaviors. In fact, in one of the earliest equivalence studies, Sidman and Cresson (1973) worked with a retarded youth who could match spoken words to pictures, and could vocally name the pictures, but was unable to match the printed words to the dictated names. However, after the youth was trained to match the printed words to the pictures, he was able to select any set of 20 pictures or picture names conditionally upon a dictated name. Furthermore, given a printed name sample, he could select the corresponding picture and given a picture sample, he could select its printed name. The boy's emergent ability to perform two new sets of matching tasks demonstrated that the matching-to-sample training and test procedure had created 20,3 -member classes of equivalent stimuli.

Research on stimulus equivalence has provided researchers with a valuable means of training academic skills such as reading (e.g., Mackay, 1985, Sidman, 1971) and spelling (e.g., Stromer, Mackay, \& Stoddard, 1992). For example, Mackay and Sidman (1984) and Mackay (1985) found that equivalence classes involving printed words could be established by teaching subjects to construct the printed words with movable letters. In their studies, Makay and Sidman (1984) taught students who were mentally retarded to name color patches and construct the color names using movable letters. Students subsequently matched printed-word comparisons to color patches and color comparisons to printed-word samples, and also named printed words.

Although the applied potential of stimulus equivalence research has been noted, the actual use of equivalence research in the applied setting has been very limited (see Mace, 1994; Sidman, 1994), although there are some recent exceptions. In one study, for example, Lynch and Cuvo 
(1991) used the matching-to-sample training procedure to teach fraction and decimal equivalence to 7 fifth and sixth grade students, who were identified by their math teachers as having difficulty on these tasks despite formal instruction. The students were taught to match pictorial representations of fractions (B comparison stimuli), to printed counterpart fraction ratios (A sample stimuli), and to match printed decimals (C comparison stimuli), to pictorial representations of fractions (B sample stimuli). For example, the three stimuli in a class may have been as follows; $1 / 5$ (a), pictorial analogue of $1 / 5$ (b), and 0.20 (c). Posttest performance by all subjects indicated the emergence of equivalence relations between stimuli, but subjects only showed low levels of generalization. Generalization tests involved relating printed fractionprinted decimal and printed decimal-printed fraction relations to novel sample and comparison quantities following training and posttest trials.

Although equivalence has made only a relatively small impact on applied behavior analysis, further research in this respect should be driven, in part, by the results of basic research. In particular, any studies that have identified methods for facilitating equivalence class formation should be of interest to applied workers. One relevant example involved the use a respondent-type ${ }^{1}$ procedure, rather than matching-to-sample training (Leader, Barnes, \& Smeets, 1996). During the training, nine nonsense syllables were presented to the subject in the form of six stimulus pairs. The first stimulus of each pair simply appeared on the screen for $1 \mathrm{~s}$ (e.g., A1). The screen subsequently cleared for $0.5 \mathrm{~s}$ (within pair delay) before the second stimulus of the pair (i.e., B1) appeared for 1 s. A 3-s interval (between pair delay) then occurred before the next stimulus pair was presented in the same fashion. All six stimulus pairs $(\mathrm{A} 1 \rightarrow \mathrm{B} 1, \mathrm{~A} 2 \rightarrow \mathrm{B} 2, \mathrm{~A} 3 \rightarrow \mathrm{B} 3, \mathrm{~B} 1 \rightarrow \mathrm{C} 1, \mathrm{~B} 2 \rightarrow \mathrm{C} 2, \mathrm{~B} 3 \rightarrow \mathrm{C} 3)$ were presented in this way in a quasirandom order for 60 trials, the only constraint being that each stimulus pair was presented once in each successive block of 6 trials (i.e., each stimulus pair was presented 10 times). When all stimulus pairs were presented, subjects were tested for the emergence of symmetry and equivalence relations using a standard matching-tosample test. The vast majority of subjects successfully passed the equivalence test $(84 \%)$.

In another study, Smeets, Leader, and Barnes (1997) investigated whether the respondent procedure (e.g., $A \rightarrow B, B \rightarrow C$ ) would produce symmetry and equivalence formation in 5-year-old normal functioning children. In Experiments 3 and 4 of this study, when a simple-to-complex protocol was employed (train A-B test B-A, train B-C test C-B), $87.5 \%$ of

\footnotetext{
${ }^{1}$ Consistent with our previous publications in this area, we have included the suffix "type" to indicate that the respondent training procedure described in this article differs considerably from traditional respondent conditioning experiments. For example, the respondent-type training procedure presents nine conditional stimuli (i.e., nonsense syllables) in various sequences, whereas a typical respondent conditioning experiment presents one or two conditional stimuli and an unconditioned stimulus. We would like to stress that using the term respondent-type does not imply that the main behavioral process produced by this procedure is best characterized as respondent behavior.
} 
subjects matched all directly paired stimuli (B-A and C-B) and $76.2 \%$ of subjects matched all indirectly paired stimuli (C-A). Given that the respondent-type training procedure appears to work very well with both adults and children, it may be useful to explore its potential in the applied domain. This may be particularly important given that we have gathered some data that indicates that the respondent training procedure may be somewhat more effective in producing equivalence responding than the traditional matching-to-sample preparation (Leader \& Barnes-Holmes, in press).

The purpose of the present study was to determine whether the respondent-type training procedure could be used to establish fraction and decimal equivalencies in 5-year-old children. Furthermore, this study sought to investigate emergent generalization based on physical similarity (Barnes \& Keenan, 1993), rather than mathematical generalization (as used by Lynch \& Cuvo, 1991). Generalization tests involved presenting subjects with stimuli seen in training as samples and a series of shapes that are physically similar (but different from those seen in training) as comparisons. The intention was not to replicate the study of Lynch and Cuvo but to investigate generalization on the basis of physical similarity. This was deemed important because, the expansion of equivalence classes based on physical similarity has not yet been shown in young children. Furthermore, it seems likely that responding based on physical quantities is a prerequisite to mathematical understanding (Hayes, Barnes-Holmes \& Roche, in press), and thus a thorough analysis of equivalence class expansion based on stimulus generalization in young children seems important. The current study consisted of three experiments. In Experiment 1 subjects were given conditional discrimination training using fractions and decimals as stimuli and were then tested for the emergence of equivalence classes. Experiment 2 was identical to Experiment 1, except that subjects were also administered three generalization tests that examined generalization between mathematically equivalent stimuli and physically similar stimuli. Experiment 3 was identical to Experiment 2, except that subjects were given an additional generalization test.

\section{General Method}

\section{Subjects}

Twenty four normal functioning 5-year-old Dutch preschool children served as subjects. The children were randomly assigned to each of the three experiments ( 8 in each experiment). Age (years and months) and sex of subjects are shown in Tables 1, 2, and 3.

\section{Apparatus}

The stimuli $(3.0 \times 3.0 \mathrm{~cm})$ consisted of squares, circles, decimals, and fractions (see Figure 1) and are indicated by alphanumerical codes (e.g., $\mathrm{X} 1, \mathrm{A1})$. The stimuli were presented on white cards $(14.5 \times 20.0 \mathrm{~cm})$ covered with plastic to prevent staining. Observation cards displayed one stimulus (e.g., B1) positioned at the center of the card. Matching cards displayed three stimuli, two horizontally aligned (e.g., C1 and C2) $9 \mathrm{~cm}$ 
$\begin{array}{llll}\mathrm{X} 1 & \mathrm{X} 2 & \mathrm{Y} 1 & \mathrm{Y} 2\end{array}$

(a) $\# \quad \%$

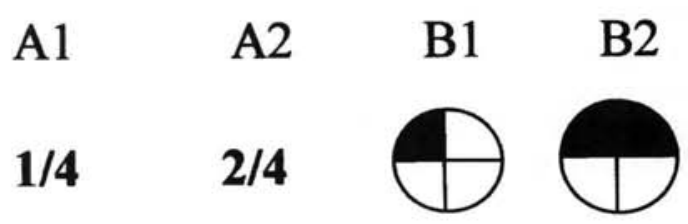
C1
$\mathrm{C} 2$
0.25
0.50

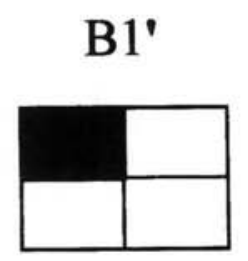

B1"

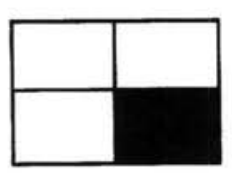

B2'

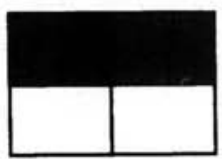

B2"

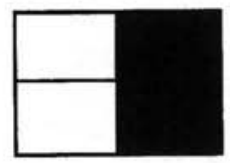

Figure 1. Fractions, decimals, and shapes used as stimuli.

apart, and one stimulus centered $3 \mathrm{~cm}$ below (e.g., A2). Additional materials were a tray with beads and a standing transparent glass tube displaying a mark. Filling the tube to the mark required 50 beads. 


\section{Sessions and Setting}

Sessions were conducted in a quiet room in the children's school building, once a day, 5 days a week, typically for a duration of 10 to $15 \mathrm{~min}$. Participating in the study were 3 adults, 1 as experimenter, and 2 as reliability observers. One of 2 reliability observers was present in the same room but was situated so that she or he could clearly observe the stimuli and the subjects' responses, but not the experimenter's data sheet.

\section{Trials, Responses, Recordings, and Contingencies}

Three types of trials were used: respondent training trials, matching-tosample training trials, and matching-to-sample test trials. During a respondent training trial, the experimenter showed the child an observation card (e.g., A1) for $1 \mathrm{~s}$ (the experimenter silently counted " 21 ") followed by another card (e.g., C1) for $1 \mathrm{~s}$. The experimenter then recorded whether the subject had looked at both observation cards and without delivering any programmed consequences initiated a 3-s interval before commencing the next training trial (i.e., there was a 3-s delay between the presentation of one stimulus pair and the next). If after every fifth training trial the subject had observed all stimulus presentations, the experimenter praised the subject (i.e., "Good girl/boy, you are doing well, take a bead.").

Two types of matching-to-sample training trials were used: demonstration and no-help trials. A demonstration trial began with the experimenter presenting a matching card and pointing to the sample and designating the correct comparison saying, "If you see this, point to that." The experimenter then requested the subject to point to the sample and correct comparison. During the matching-to-sample no-help trials, the subject did not receive instruction or modeling - the experimenter simply presented the matching card to the subject without any comment. Responses on the matching-to-sample training trials were scored as correct, incorrect, or invalid. Correct or incorrect responses were defined as pointing to the correct or incorrect comparison, and invalid responses were recorded when the subject pointed, for example, to the comparison without looking at the sample. Correct responses were followed by verbal praise and the delivery of a token ("Good, take a bead"). Incorrect responses were followed by verbal disapproval ("wrong, no bead"), and invalid responses were followed by corrective feedback (e.g.,"look at the picture when pointing").

Matching-to-sample test trials were the same as the no-help trials (silent presentation of matching cards) except that responses consistent with the matching-to-sample training were simply recorded and no programmed consequences were delivered. In addition to the nonverbal responses (pointing to the comparison stimuli), the experimenter also recorded the subjects' verbal comments on the respondent training and matching-to-sample training and test trials. 


\section{Experiment 1}

\section{Procedure}

Step 1A: Pretraining X-Y. This step was designed to establish an unrelated conditional discrimination with abstract $X$ and $Y$ stimuli (see Figure 1), so that any "failures" on the matching-to-sample test with decimal and fraction stimuli could not be attributed to unfamiliarity with the task. One block of 18 matching-to-sample training trials was used. The step began with two demonstration trials in which $\mathrm{X} 1$ and $\mathrm{X} 2$ served as samples and $Y 1$ and $Y 2$ as comparisons. This was followed by 16 no-help trials. In this step the $Y 1$ and $Y 2$ comparisons were always placed in the same position (i.e., $\mathrm{Y} 1$ to the left and $\mathrm{Y} 2$ to the right). A stability criterion of 15 out of 16 correct responses $(94 \%)$ on the no-help trials was required and subjects were allowed two exposures to the stage.

Step 1B: This step consisted of 16 no-help trials. The step was identical to Step $1 \mathrm{~A}$, except that no demonstration trials were used and the position of the comparison stimuli was reversed (i.e., $\mathrm{Y} 1$ to the right and $\mathrm{Y} 2$ to the left).

Step 1C: In this step 16 no-help trials were used. The position of the comparisons was randomized. That is, 8 trials with $\mathrm{Y} 1$ to the right and $\mathrm{Y} 2$ to the left quasirandomly mixed with 8 trials during which the stimulus positions were reversed.

Step 2: Testing $X-Y$ and $Y-X$. This step examined whether the subjects continued the $X-Y$ performance accurately: (a) without programmed consequences, and (b) when the sample-comparison functions of the $X$ and $Y$ stimuli were reversed. The step consisted of two blocks of test trials (Blocks 1 \& 3) and two blocks of training trials (Blocks 2 \& 4). Each training block consisted of $6 \mathrm{X}-\mathrm{Y}$ training trials and each test block consisted of $4 \mathrm{X}-\mathrm{Y}$ trials quasirandomly mixed with $4 \mathrm{Y}-\mathrm{X}$ trials.

Prior to the commencement of a test block, the experimenter removed the tray of beads and glass tube and said, "Now we are going to play the game without me telling you whether you are right or wrong. You won't get any beads. Later on we will play the game with beads. Do your best." The experimenter then proceeded with the test trial and refrained from any communication with the subject. Prior to the commencement of a training block, the experimenter placed the tray of beads and the glass tube on the table and said "Now you can earn beads again." When the subject succeeded in filling the glass tube to the mark ( 50 beads) during this stage or any other stage of the experiment, the subject was allowed to exchange the beads for a preselected card (cartoon character, soccer player, animal). A stability criterion of (a) $7 / 8$ correct responses $(87.5 \%)$ on $X-Y$ test trials, (b) $7 / 8$ correct responses on the $Y-X$ test trials, and (c) $11 / 12$ correct responses $(92 \%)$ on the $X-Y$ training trials was required to proceed to the next stage of the experiment. Subjects were allowed two exposures to this stage.

Step 3: Train A-B, Test B-A. In this step subjects received respondent training during which they observed the Stimuli $A \rightarrow B$ in a fixed temporal 
order. This was followed directly by the conditional discrimination probes $B-A$. During $A 1 \rightarrow B 1$ respondent training trials subjects were presented with $1 / 4$ (A1) followed by a circle divided into four quarters with the upper left quarter shaded (B1). During $A 2 \rightarrow B 2$ respondent training trials subjects were presented with $2 / 4$ (A2) followed by a circle divided into four quarters with the upper two quarters shaded (B2) (see Figure 1).

Step 3 consisted of six blocks. In the first block subjects received 10 $\mathrm{A} \rightarrow \mathrm{B}$ respondent training trials. Block 2 consisted of $1 \mathrm{X}-\mathrm{Y}$ and $1 \mathrm{Y}-\mathrm{X}$ matching-to-sample test trial followed by $8 \mathrm{~B}-\mathrm{A}$ conditional discrimination probes. Block 3 consisted of $6 X-Y$ training trials. Blocks 4,5 , and 6 were identical to Blocks 1,2 , and 3 respectively. Subjects reached the mastery criterion if they observed the $A \rightarrow B$ stimulus presentation $18 / 20$ times $(90 \%)$ during the respondent training trials (Blocks $1 \& 4$ ), produced 14/16 correct responses $(87.5 \%)$ on the $B \rightarrow A$ test trials, produced $3 / 4$ correct responses $(75 \%)$ on the $X-Y$ and $Y-X$ test trials (Blocks $2 \& 5$ ), and produced $11 / 12$ correct responses $(92 \%)$ on the $X-Y$ training trials (Blocks $3 \& 6)$. Subjects were allowed two exposures to this stage.

Step 4: Train C-B, Test B-C. This step was identical to Step 3 except that (a) each respondent training block consisted of $10 \mathrm{C} \rightarrow \mathrm{B}$ respondent training trials, and (b) Blocks 2 and 4 consisted of $1 \mathrm{X}-\mathrm{Y}, 1 \mathrm{Y}-\mathrm{X}, 8 \mathrm{~B}-\mathrm{C}$, and $2 \mathrm{~B}-\mathrm{A}$ test trials. During $\mathrm{C} 1 \rightarrow \mathrm{B} 1$ respondent training trials subjects were presented with 0.25 (C1) followed by a circle divided into four quarters with the upper left quarter shaded (B1). During $\mathrm{C} 2 \rightarrow \mathrm{B} 2$ respondent training trials subjects were presented with $0.50(\mathrm{C} 2)$ followed by a circle divided into four quarters with the upper two quarters shaded (B2) (see Figure 1).

Step 5: Test A-C and C-A. This step determined whether subjects matched both directly and indirectly paired stimuli. This step consisted of six blocks. Block 1 consisted of $1 \mathrm{X}-\mathrm{Y}, 1 \mathrm{Y}-\mathrm{X}$, and $4 \mathrm{~B}-\mathrm{A}$ test trials quasirandomly mixed with 4 B-C test trials. Block 2 consisted of 4 A-C test trials quasirandomly mixed with $4 \mathrm{C}-\mathrm{A}$ test trials. Block 3 consisted of $6 \mathrm{X}-\mathrm{Y}$ training trials. Blocks 4,5 , and 6 were identical to Blocks 1,2 , and 3 respectively. Subjects reached the mastery criterion if they (a) produced $3 / 4$ correct responses $(75 \%)$ on the $X-Y$ and $Y$-X test probes, $7 / 8$ correct responses $(87.5 \%)$ on the B-A test probes, and $7 / 8$ correct responses on the B-C test probes (Blocks $1 \&$ \&), and (b) produced 14/16 correct responses $(87.5 \%)$ on the A-C and C-A test trials (Blocks 2 \& 4) and produced 11/12 correct responses (92\%) on the $X-Y$ training trials (Blocks $3 \& 6)$.

\section{Reliability}

Reliability checks were carried out on $30 \%$ of all training and test trials. Experimenter and observer disagreed on one matching-to-sample test trial.

\section{Results and Discussion}

Percent correct on training and test trials are presented in Table 1. All 8 subjects in Experiment 1 required one block of training trials in Steps $1 \mathrm{~A}, 1 \mathrm{~B}$, and $1 \mathrm{C}$ to establish the pretrained $\mathrm{X}-\mathrm{Y}$ conditional relations, and 
Table 1

Sex, Age, and Correct Responses on Training and Test Trials in Experiment 1

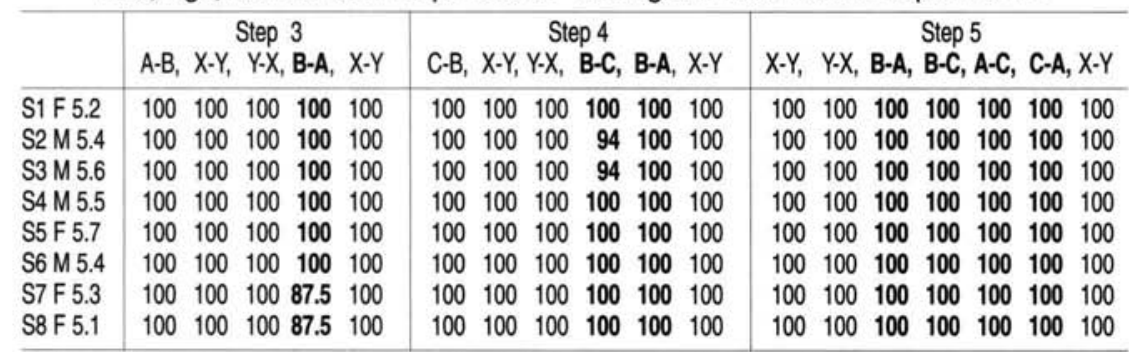

Note. Plain text denotes percent correct during training trials and bold text denotes percent correct during test trials.

they maintained this performance throughout the experiment. In Step 2, all subjects reached criterion performance after one block of $X-Y$ and $Y-X$ test trials. In Step 3, Subjects 7 and 8 produced $87.5 \%$ correct responding on the B-A test probes, and the remainder of subjects achieved $100 \%$ correct responding. In Step 4, Subjects 2 and 3 produced $94 \%$ correct responding on the $\mathrm{B}-\mathrm{C}$ test probes, and the remainder of subjects attained $100 \%$ correct responding. In Step 5, subjects were successful on all test probes.

This experiment demonstrated that 5-year-old children are capable of forming equivalence classes using fractions, decimals, and pictorial representations as stimuli. Experiment 2 was designed to determine whether these findings could be replicated, and to establish whether subjects would show generalization with $1 / 4,2 / 4,0.25$, and 0.50 as samples and novel shapes as comparisons.

\section{Experiment 2}

\section{Procedure}

Steps 1 through 5 were identical to Experiment 1.

Generalization Test No.1: The Stimuli A1, A2, C1, and C2 (i.e., 1/4, $2 / 4,0.25$, and 0.50 ) served as samples. Shapes not seen in training, but with the same shaded areas, served as comparison stimuli. That is, when subjects were presented with B1' they observed a square divided into four quarters with the upper left quarter shaded, and when B2' was presented, they observed a square divided into four quarters with the upper two quarters shaded (see Figure 1). This generalization test consisted of four blocks. Block 1 consisted of 4 B1'-A1, 4 B2'-A2, 4 B1'-C1, and 4 B2'-C2 test trials quasirandomly mixed. Block 2 consisted of $6 \mathrm{X}-\mathrm{Y}$ training trials. Blocks 3 and 4 were identical to Blocks 1 and 2 respectively. The mastery criterion consisted of a minimum of $30 / 32$ correct responses $(94 \%)$ across the two test blocks (Blocks 1 and 3 ) and 11/12 correct responses $(92 \%)$ on the training trials (Blocks 2 and 4 ). 
Generalization No. 2: Generalization Test No. 2 was identical to the previous one, except that the comparison stimuli were altered, in that a different area of the shapes was shaded (i.e., B1" consisted of a square divided into four quarters with the lower right quarter shaded, and B2" consisted of a square divided into four quarters with the upper and lower right quarters shaded (see Figure 1).

Generalization Test No. 3: This generalization test was identical to the previous two tests, except that the comparison stimuli were altered once again in that the number of shaded and unshaded areas was increased. That is, B1"' consisted of a square divided into 16 segments with segments $1,8,10$, and 16 shaded, and B2'" consisted of a square divided into 16 segments with segments $1,3,6,10,12,13,15$, and 16 shaded (see Figure 2).

\section{Reliability}

Reliability checks were carried out on $35 \%$ of all training and test trials. Experimenter and reliability observer always agreed.

B1"'

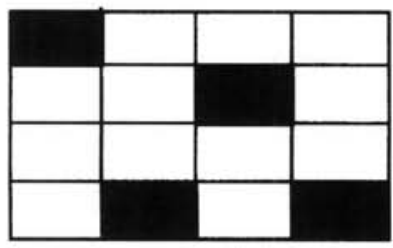

B2"'

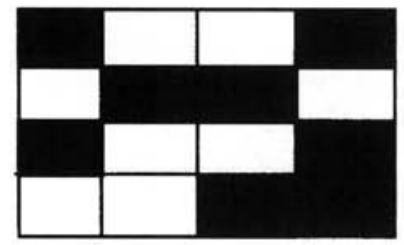

B1"'

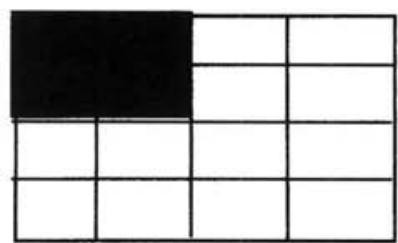

B2"'"

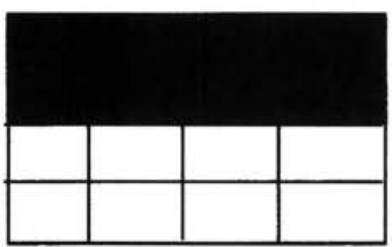

Figure 2. Fractions, decimals, and shapes used as stimuli. 


\section{Results and Discussion}

Percent correct on training and test trials are presented in Table 2. All 8 subjects in Experiment 2 required one block of training trials in Steps $1 \mathrm{~A}, 1 \mathrm{~B}$, and $1 \mathrm{C}$ to establish the pretrained $\mathrm{X}-\mathrm{Y}$ conditional relations; they maintained this performance throughout the experiment. In Step 2, all subjects reached criterion performance after one block of $X-Y$ and $Y-X$ test trials. In Step 3, all 8 subjects produced $100 \%$ correct responding on

Table 2

Sex, Age, and Percent Correct on Training and Test Trials in Experiment 2

\begin{tabular}{|c|c|c|c|c|}
\hline & & & & Generaliza \\
\hline & $A-B$, & $C-B, X-Y, Y$ & $X-Y, Y-X$ & 123 \\
\hline & 0100 & 10 & 100 & 1001 \\
\hline & & & & \\
\hline & & 100 & & \\
\hline & & 100 & 100 & \\
\hline & 100 & 100 & & 10 \\
\hline & 10 & 100 & & 100100 \\
\hline & 100 & 100 & & \\
\hline & 10 & 1001001 & 100 & \\
\hline
\end{tabular}

Note. Plain text denotes percent correct during training trials and bold text denotes percent correct during test trials.

the B-A test probes. In Step 4, Subjects 9 and 10 produced $94 \%$ correct responses on the B-C test probes, and the remainder of subjects achieved $100 \%$ correct responding. In Step 5, subjects were successful on all test probes on their first exposure to the test. All 8 subjects were successful on Generalization Tests 1 and 2, after one exposure. Subjects $9,10,11$, and 12 were successful on Generalization Test 3 after one exposure, while Subjects $13,14,15$, and 16 failed to reached the mastery criterion after the maximum of two exposures. This experiment demonstrated, as did Experiment 1, that young children show the emergence of equivalence relations between fractions, decimals, and pictorial representations. However, only $50 \%$ of subjects passed all three generalization tests. Experiment 3 was designed to determine whether administering an extra generalization test would improve performance on Generalization Test 3.

\section{Experiment 3}

\section{Procedure}

Training and testing of Steps 1 through 5 and Generalization Tests 1 and 2 were identical to Experiment 2.

Generalization Test No. 3: Generalization Test No. 3 was identical to Test 3 from the previous experiment, except that the shaded segments were contiguous with one another. That is, the B1 '"' stimulus consisted of a square divided into 16 segments with the upper left 4 segments shaded, 
and B2'"' consisted of a square divided into 16 segments with the upper 8 segments shaded (see Figure 2).

Generalization Test No. 4: Generalization Test No. 4 was identical to Generalization Test No. 3 of Experiment 2.

\section{Reliability}

Reliability checks were made on $30 \%$ of all training and test trials. Experimenter and observer always agreed.

\section{Results and Discussion}

Percent correct on training and test trials are presented in Table 3. All 8 subjects in Experiment 3 required one block of training trials in Steps

Table 3

Sex, Age, and Percent Correct on Training and Test Trials in Experiment 3

\begin{tabular}{|c|c|c|c|c|}
\hline & & & & Generalization \\
\hline & $A-B$ & C-B, & $X-Y, Y-X$, & $\begin{array}{llll}1 & 2 & 3 & 4\end{array}$ \\
\hline & 1 & 0 & 100 & 100100100 \\
\hline & & & & \\
\hline & & & & \\
\hline & & 0 & 100 & 100 \\
\hline & 0 & 0 & 100 & 100100100 \\
\hline & & 100100 & & \\
\hline & & & & \\
\hline & 10 & 00 & 100 & 100 \\
\hline
\end{tabular}

Note. Plain text denotes percent correct during training trials and bold text denotes percent correct during test trials.

$1 \mathrm{~A}, 1 \mathrm{~B}$, and $1 \mathrm{C}$ to establish the pretrained $\mathrm{X}-\mathrm{Y}$ conditional relations, and they maintained this performance throughout the experiment. In Step 2, all subjects reached the criterion performance after one block of $X-Y$ and $\mathrm{Y}-\mathrm{X}$ test trials.

In Steps 3, 4, and 5 subjects were successful on all test probes on their first exposure. Similarly, subjects were successful on all generalization tests on their first exposure.

\section{General Discussion}

This study successfully used the respondent-type training procedure to establish equivalence relations among fractions, decimals, and pictorial representations of these numerical properties, with 5-year-old children in an educational setting. In Experiment 1, all subjects successfully matched fractions to counterpart decimal ratios $(\mathrm{A}-\mathrm{C})$ and decimals to equivalent fraction ratios $(\mathrm{C}-\mathrm{A})$ in accordance equivalence relations.

The purpose of Experiment 2 was to replicate the findings of Experiment 1 and test for emergent generalization to novel pictorial representations of the fraction/decimal stimuli. As in Experiment 1, 
subjects reliably formed decimal and fraction equivalence relations. During Generalization Test 1 , subjects were presented with a shape not seen in training but the shaded areas remained the same. In Generalization Test 2, subjects were presented with a shape not seen in training and the shaded areas were altered. In Generalization Test 3, subjects were presented with a shape not seen in training, and the number of shaded and unshaded segments was increased, and the position of the shaded areas was randomized. All subjects passed the first two generalization tests, but only 4 of the 8 subjects were successful on Generalization Test 3.

In Experiment 3, subjects were administered an extra generalization test (B1'"'\& B2'"') to determine whether this would improve performance on Generalization Test 4 (Test 3 from Experiment 2). This test was identical to Test 3 in Experiment 2, but the position of the shaded areas was consistent with the training. All subjects passed this test, and then successfully passed the final test in which the position of the shaded areas was randomized. In effect, performance on Generalization Test 4 was dramatically improved when subjects were presented with a series of generalization tests that involved a gradual (rather than abrupt) progression from stimuli seen in training to novel stimuli presented during the generalization tests.

The results of Experiment 3 demonstrated that children of a young age are capable of not only demonstrating equivalence between fraction and decimal stimuli, but also of showing stimulus generalization involving novel pictorial representations that are physically similar. This is an apparently important behavioral effect because it may be critical for the subsequent demonstration of mathematical responding involving mathematically equivalent, but perceptually different, stimuli, like those employed by Lynch and Cuvo (1991). Indeed, it could be argued that until subjects pass generalization tests like those employed by Lynch and Cuvo, the experimental equivalence classes generated in such studies do not possess mathematical properties beyond the basic equivalence relations, thereby failing to provide evidence for "genuine" mathematical behavior (see Baroody \& Hume, 1991; Bell, 1991). In taking this position, however, the question then arises as to how one might generate such "genuine" mathematical properties. In addressing this question, the systematic analysis of the interaction between equivalence classes and primary stimulus generalization would seem to be an important starting point. Now that this basic interaction has been demonstrated in the current study, future research might examine how to establish appropriate responding to the proportional relations between elements of fractions and pictorial representations of those fractions (e.g., to what extent is equivalence and stimulus generalization involved in relating $1 / 4$ and $2 / 8$ to a square frame containing 4 small black squares out of a total of 16 white squares). Clearly, this is an important question for the teaching of complex and advanced mathematical skills.

One criticism of the current study might be that the mathematical stimuli 
employed allowed the subjects to respond to specific elements of the stimuli. For example, subjects may have discriminated the two fractions, based on the numerators, 1 and 2 . The same criticism may be made regarding the decimals $(0.25,0.50)$ with 2 and 5 being the discriminative elements. Even the generalization tests could be criticized on the basis that subjects discriminated physical similarity based on "more or less black." An important follow-up study, therefore, would involve using 1/4, 2/4, 4/1, 4/2 and their corresponding decimal and pictorial representations. This study would allow one to determine whether subjects discriminated stimuli on the basis of complex stimuli with discriminative elements or on the basis of "genuine" mathematical comprehension.

The respondent-type training procedure is a relatively new nonmatching-to-sample training procedure. This study represents an important advance in that it demonstrated the effectiveness of a new procedure in the applied setting. Given these findings it may now be possible to use the respondent procedure in other areas of research in the applied domain. One possible avenue for future research would be to replicate the Lynch and Cuvo study, but to use the respondent training procedure. This would allow us to determine whether the alternative methodology improves older subjects' fraction-decimal equivalence class formation, and whether it has any effect on the generalization test performances. Although subjects in the Lynch and Cuvo study were older than subjects in the present study (subjects were aged between 11 to 13 years of age) and the range of stimuli used were more complex, subjects only showed low to moderate levels of generalization. It was demonstrated in this study that successful generalization may require more gradual fading to increasingly novel stimuli. Subjects in the current study were successful across all generalization tests after the difference from one test to the next involved a gradual shift along the dimension toward more novel pictorial stimuli. Perhaps a similar approach could be adapted with the Lynch and Cuvo procedures. In effect the presentation of fraction and decimal ratios familiar to the student with their counterpart pictorial representations followed by fraction and decimal ratios unfamiliar to the student and their pictorial representations may allow for greater success on subsequent generalization tests with novel fraction and decimal stimuli.

In conclusion, the respondent training procedure was clearly an effective means of teaching new decimal and fraction relations via the equivalence paradigm. In the classroom setting, however, difficulties may arise in maintaining the student's attention. Obviously, the materials used in this experimental study would have to be modified to accommodate such difficulties. Nevertheless, the basic procedure is effective, and thus warrants further attention within the applied domain. 


\section{References}

BARNES, D. (1994). Stimulus equivalence and relational frame theory. The Psychological Record, 44, 91-124.

BARNES, D., \& KEENAN, M. (1993). A transfer of functions through derived arbitary and non-arbitary stimulus relations. Journal of the Experimental Analysis of Behaviour, 59, 61-81.

BAROODY, A. J., \& HUME, J. (1991). Meaningful mathematics instruction: The case of fractions. Remedial and Special Education, 12, 54-68.

BELL, A. (1993). Principles for the design of teaching. Educational Studies in Mathematics, 24, 5-34.

FIELDS, L., \& VERHAVE, T. (1987). The structure of equivalence classes. Journal of the Experimental Analysis of Behavior, 48, 317-332.

HAYES, S. C. (1991). A relational control theory of stimulus equivalence. In L. J. Hayes \& P. N. Chase (Eds.), Dialogues of verbal behavior (pp.19-40). Reno, NV: Context Press.

HAYES, S. C., BARNES-HOLMES, D., \& ROCHE, B. (in press). A post-Skinnerian analysis of human language and cognition. New York: Plenum.

LEADER, G., \& BARNES-HOLMES, D. (in press). Matching-to-sample and respondent-type training as methods for producing equivalence relations: Isolating the critical variable. The Psychological Record.

LEADER, G., BARNES, D., \& SMEETS, P. M. (1996). Establishing equivalence relations using a respondent-type training procedure. The Psychological Record, 46, 685-706.

LYNCH, D. C., \& CUVO, A. J. (1991). Stimulus equivalence instructions of fractiondecimal relations. Journal of Applied Behavior Analysis, 28, 115-126.

MACE, F. C. (1994). Basic research needed for stimulating the development of behavioral technologies. Journal of the Experimental Analysis of Behavior, 61, 529-550.

MACKAY, H. A. (1985). Stimulus equivalence in rudimentary reading and spelling. Analysis and Intervention in Development Disabilities, 5, 373-387.

MACKAY, H. A., \& SIDMAN, M. (1984). Teaching new behaviours via equivalence relations. In P. H. Brooks, R. Sperber, \& C. MacCauley (Eds.), Learning and cognition in the mentally retarded (pp. 493-513). Hillsdale, NJ: Erlbaum.

SIDMAN, M. (1971). Reading and auditory-visual equivalence. Journal of Speech and Hearing Research, 14, 5-13.

SIDMAN, M. (1980). A note on the measurement of conditional discrimination. Journal of the Experimental Analysis of Behavior, 33, 285-289.

SIDMAN, M. (1986). Functional analysis of emergent and verbal classes. In T. Thompson \& M. A. Zeiler (Eds.), Analysis and interaction of behavioral units (pp. 213-245). Hillsdale, NJ: Erlbaum.

SIDMAN, M. (1987). Two choices are not enough. Behavior Analysis, 22, 11-18.

SIDMAN, M. (1994). Equivalence relations: $A$ research story. Boston, MA: Authors Cooperative.

SIDMAN, M., \& CRESSON, O., Jr. (1973). Reading and crossmodal transfer of stimulus equivalence in severe retardation. American Journal of Mental Deficiency, 77, 513-523

SMEETS, P. M., LEADER, G., \& BARNES, D. (1997). Establishing stimulus classes in adults and children using a respondent-type training procedure: A follow-up study. The Psychological Record, 47, 285-308.

STROMER, R., MACKAY, H. A., \& STODDARD, L. T. (1992). Classroom applications of stimulus equivalence technology. Journal of Behavioral Education, 2, 225-256. 
